\title{
In-room ultrasound fusion combined with fully compatible 3D-printed holding arm - rethinking interventional MRI
}

This article was published in the following Dove Press journal:

Medical Devices: Evidence and Research

\section{Michael Friebe,' Juan \\ Sanchez,' Sathish \\ Balakrishnan,' Alfredo \\ Illanes,' Yeshaswini Nagaraj, ${ }^{2}$ \\ Robert Odenbach,' \\ Marwah Matooq,' Gabriele \\ Krombach, ${ }^{3}$ Michael Vogele, ${ }^{4}$ \\ Axel Boese' \\ 'Chair of Intelligent Catheter, Otto- von-Guericke-University, Magdeburg, Germany; ${ }^{2}$ University of Groningen, University Medical Center Groningen, Center for Medical Imaging North East Netherlands, Groningen, the Netherlands; ${ }^{3}$ Universitätsklinikum Giessen, Radiologische Klinik, Giessen, Germany; ${ }^{4}$ Interventional Systems $\mathrm{GmbH}$, Kitzbühel, Austria \\ Video abstract

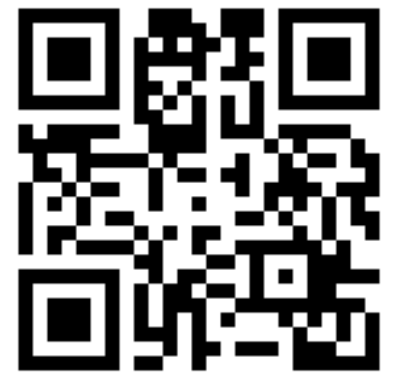 \\ Point your SmartPhone at the code above. If you have a QR code reader the video abstract will appear. Or use: http://youtu.be/p6W9v0-DIIO}

Correspondence: Michael Friebe Chair of Intelligent Catheters and Image Guided Surgeries, Faculty of Electrical Engineering, Institute of Medical Technology, Otto-von-Guericke-University, Rötgerstr. 9, D-39104 Magdeburg, Germany Email michael.friebe@ovgu.de

\begin{abstract}
There is no real need to discuss the potential advantages - mainly the excellent soft tissue contrast, nonionizing radiation, flow, and molecular information - of magnetic resonance imaging (MRI) as an intraoperative diagnosis and therapy system particularly for neurological applications and oncological therapies. Difficult patient access in conventional horizontal-field superconductive magnets, very high investment and operational expenses, and the need for special nonferromagnetic therapy tools have however prevented the widespread use of MRI as imaging and guidance tool for therapy purposes. The interventional use of MRI systems follows for the last 20+ years the strategy to use standard diagnostic systems and add more or less complicated and expensive components (eg, MRI-compatible robotic systems, specially shielded in-room monitors, dedicated tools and devices made from low-susceptibility materials, etc) to overcome the difficulties in the therapy process. We are proposing to rethink that approach using an in-room portable ultrasound (US) system that can be safely operated till $1 \mathrm{~m}$ away from the opening of a $3 \mathrm{~T}$ imaging system. The live US images can be tracked using an optical inside-out approach adding a camera to the US probe in combination with optical reference markers to allow direct fusion with the MRI images inside the MRI suite. This leads to a comfortable US-guided intervention and excellent patient access directly on the MRI patient bed. This was combined with an entirely mechanical MRI-compatible 7 degrees of freedom holding arm concept, which shows that this test environment is a different way to create a costefficient and effective setup that combines the advantages of MRI and US by largely avoiding the drawbacks of current interventional MRI concepts.
\end{abstract}

Keywords: interventional MRI, fusion imaging, ultrasound/MRI hybrid, MRI-compatible, medical holding arm ultrasound guided MRI intervention

\section{Introduction to interventional MRI}

The unparalleled soft tissue contrast and excellent image quality of magnetic resonance imaging (MRI) have made it the imaging modality of choice, especially for neurological and oncological applications. The obtained MRI images are then frequently used as relevant preoperative images for surgery planning or as a monitor-presented visual reference in the surgery suite.

MRI systems directly employed for therapeutic guidance or surgical monitoring are rarely available. The standard imaging systems in the surgery room are typically X-ray, ultrasound (US), or endoscopy (video) and are used to perform the patient monitoring, controlling, and device guiding functions.

To use these preoperative MRI images for therapy purposes as well usually requires fusion with the available imaging data in the surgery room. That is either 
not done at all or happens only in the head of the clinician, so called cognitive fusion. This requires experience and training and leads to inaccuracies, subjectivity, variability, and lack of reproducibility. ${ }^{1}$ It can also be accomplished by more or less accurate and complicated special software tools in combination with optical and/or electromagnetic device tracking systems. ${ }^{1-4}$

For the fusion via software-based image coregistration, the images are manually or semi-automatically registered to each other. A preoperative MRI image - as the wording implies - was taken before the surgery, however, sometimes even on a bed with different curvature than the surgery table or in a different patient position.

For prostate MRI, for example, the imaging is typically done with the patient in a straight supine position, while the actual biopsy or therapeutic treatment is performed with the patient prone or supine with the legs angled toward the torso.

And for breast MRI, the patient typically lies in an elevated prone position while surgery is performed on a surgical table in supine position or on the side. You easily recognize the difficulties using these preoperative MRI images for accurate image fusion with the available imaging modalities available in the therapy or surgery room.

For both procedures, there is relatively little space left in a conventional horizontal-field MRI system, not leaving maneuverable space for the surgeon or longer instruments, especially if they are orthogonal to the patient body.

Real precise image registration (US to MRI) is also not possible in this way, with typical image registration errors of the order of 3-7 mm, and in case of missing fiducial marker structures sometimes even significantly larger. That may be sufficient for actual therapies using an additional imaging system or for coarse orientation, but is too large for tissue biopsies and other precise therapies on small structures. This can be reduced to $2-3 \mathrm{~mm}$ by adjusting the MRI imaging position to the actual surgery position. ${ }^{5}$

Using real-time MRI as a guidance device for intraoperative or interventional therapies (eg, biopsies and treatment of prostate cancer, liver laser ablation/radiofrequency ablation/ or cryoablations, brain interventions) could be very beneficial and would obviously eliminate or greatly reduce these registration errors.

This requires that the therapeutic procedure is performed in the MRI suite or the MRI system is placed in a dedicated surgery room.

The latter is an option that has been explored by several institutions, but comes with very high investments and requires very highly skilled interdisciplinary staff (Figures 1 and 2). These systems are ideally suited for research institutions that explore image guidance options for developing advanced procedures, but are not an option for the large majority of clinical providers at the moment.

\section{Conventional diagnostic MRI for interventions}

Difficult patient access in conventional horizontal-field superconductive magnets and the need for special nonferromagnetic therapy tools have also prevented the widespread "occasional" interventional use of conventional diagnostic MRI systems.

This means that an MRI system that was primarily purchased and installed for diagnostic imaging purposes is also used for selected easy biopsy or needle aspiration procedures. But even these relatively simple interventions require special tools that are safe for the patient (MR safe) and do not cause significant imaging artifacts (MR compatible).

A typical artifact is caused by the susceptibility of the devices material, which can lead to a significant increase in the diameter of the device. In a high-field MRI system, an MRI-compatible needle is, for example, shown with a 10 $\mathrm{mm}$ or greater diameter, but actually only has a diameter of $2 \mathrm{~mm}$. It is obvious that with these artifacts, guidance and secure placement in smaller structures is very difficult and sometimes impossible.

Any external system that is used in or close to the MRI magnet is exposed to the strong magnetic fields, and so it must be ensured that the devices are not pulled into the magnet bore. Also, the strong electrical and magnetic fields of the MRI can lead to system malfunction or patient safety issues. But the devices itself also can influence the performance of the MRI if not properly shielded. A lot of things to consider.

The interventional and therapeutic use of MRI follows for the last $20+$ years the strategy to employ conventional standard high-field systems and add more or less complicated and expensive components. ${ }^{2}$

MRI-compatible robotic systems were, for example, proposed to solve the patient access issue in combination with specially shielded in-room monitors, dedicated nitinol or plastic devices with no or little susceptibility-related imaging artifacts, and in-room optical tracking systems. ${ }^{3}$ These components are quite expensive, and so the possible therapies are still limited because of the tight space in the MRI bore and the issues around surgical sterility. 


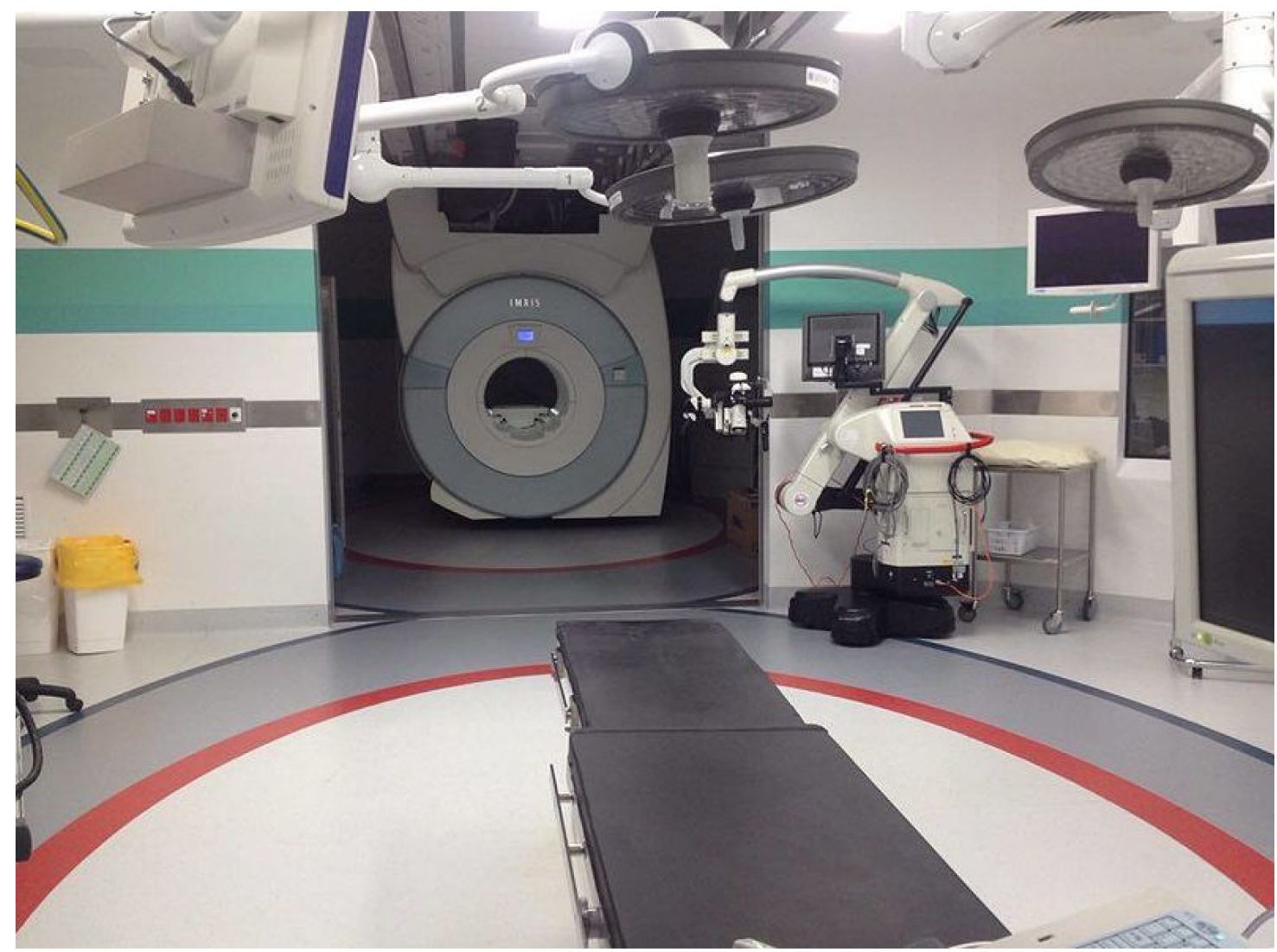

Figure I Dedicated interventional MRI setup with an MRI attached to the ceiling and parked in the back that is moved over to the surgery table when needed and subsequently moved back.

Notes: The bore diameter is still only $70 \mathrm{~cm}$ and any devices attached to the patient that are not fully MRI compatible or that extend over the available diameter need to be removed prior to imaging (Photo by SoccerNathan9 available under the Creative Commons Attribution-Share Alike 4.0 International license).

Abbreviation: MRI, magnetic resonance imaging.

The robotic systems are even needed with the short-bore magnets of the newest MRI generation. With a magnet length of $1.20 \mathrm{~m}$, it is still $60 \mathrm{~cm}$ (an arm's length) to the center of the magnet, and with a bore diameter of $70 \mathrm{~cm}$ - with a mid-size patient placed - there is only $10-15 \mathrm{~cm}$ of available vertical space for a therapy tool (Figure 3).

Open vertical-field magnets do provide some benefits for interventional procedures, but typically have only magnetic field strength of $0.2 \mathrm{~T}$ to $0.4 \mathrm{~T}$, with currently only one system over $1 \mathrm{~T}$ and another one at $1 \mathrm{~T}$ that is unfortunately phased out (Figure 2). Lower field strength comes with increased acquisition times, which is not good for therapy applications, and a lower image quality. On the other hand, the lower field strength also reduces susceptibility artifacts and comes with a much lower magnetic attraction force making the surgery generally safer. The authors do believe, however, that the low-field systems could be an excellent base for easier, dedicated procedures, especially when preoperative high-field and high-quality MRI images are used as a base of image fusion.

\section{High-field MRI with in-room US}

The difficulty of patient access in the high-field MRI systems lead to the question of whether or not it would make more sense to rethink the interventional MRI procedure by combining MRI with US inside the MRI imaging room. For many procedures, US guidance is the gold standard that could benefit from additional, near real-time MRI guidance and imaging. ${ }^{5,6}$

The general idea is to acquire comprehensive and highquality MRI images of the area of interest, move the patient out of the tight bore remaining on the MRI patient bed, and then continue with the therapy under US guidance. For further MRI images or updates, the patient is moved back into the MRI system. This may need to be done several times during the placement of the device to ensure that the target site 


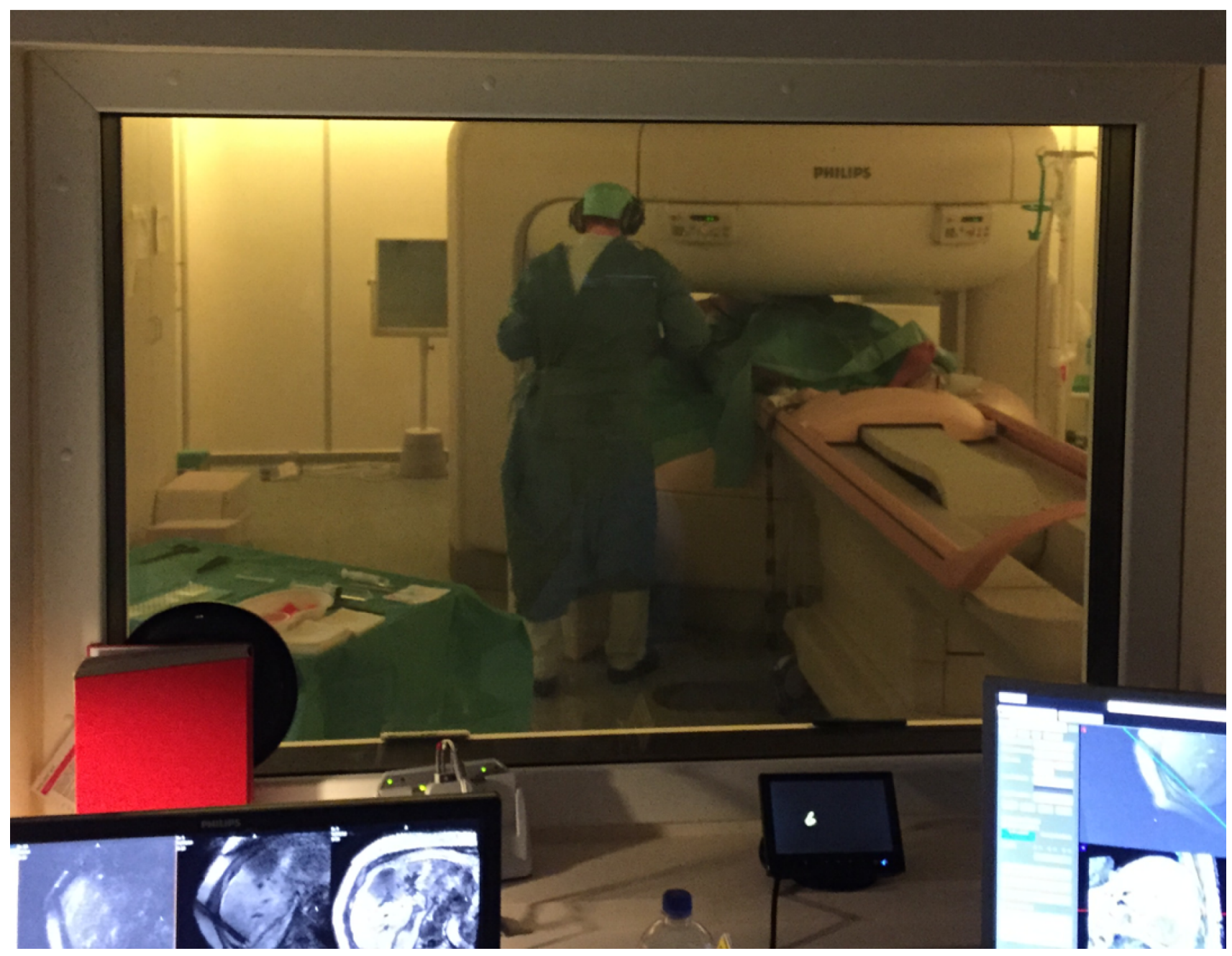

Figure 2 Open MRI used for interventional procedures - here, a radiofrequency ablation of the liver.

Notes: The vertical-field magnet allows better access to the patient especially from the side, but the available height is still limited and the surgeon has a rather uncomfortable therapy position. In the back, an in-room monitor is visible that is used for real-time guidance.

Abbreviation: MRI, magnetic resonance imaging.

has been reached and should also be done at the end of the procedure to record the therapy result for quality assurance. The MRI image is much better suited to give details on the therapy region and to show the effects of the procedure.

The images of the MRI and US are either cognitively fused (Figure 4B), ${ }^{7}$ or automatically coregistered and overlaid to the just-obtained MRI images using special tracking and navigation techniques (Figure 5). Several commercially available US systems could theoretically be used even as close as $1 \mathrm{~m}$ to a $3 \mathrm{~T}$ magnet opening (Figure 4A).

While these systems do not have an official MRI-safe label and are not tested and approved for use in an MRI imaging suite, research institutions like ours have used these systems in phantom setups to rethink interventional MRI procedures. The US system that we used (GE Venue 50) with a commercial 3T MRI (Siemens Skyra) turned itself off once it was moved too close to the magnetic field - at around 25 $\mathrm{mT}$ - but we did not experience major magnetic attraction forces even at the $50 \mathrm{mT}$ magnetic field line.

A more accurate alternative to the cognitive fusion using the MRI images and the live US images (Figure 4B) is to use a marker-based approach in combination with a navigation system that is attached to the US probe. The marker, for example, a combination of an MRI-visible component (eg, Vitamin B pills) and an optical marker, allows for registration of live US with the just acquired MRI images. ${ }^{8,9}$ This approach also has the advantage compared to the normally used outside-in optical tracking systems in that there is no or only a small issue with obstructing the line of sight between the camera and the marker and it is rather cheap and easy to use. The disadvantage is the rather small field of view, but that may not be as relevant as the area of procedure is known and limited in size. 


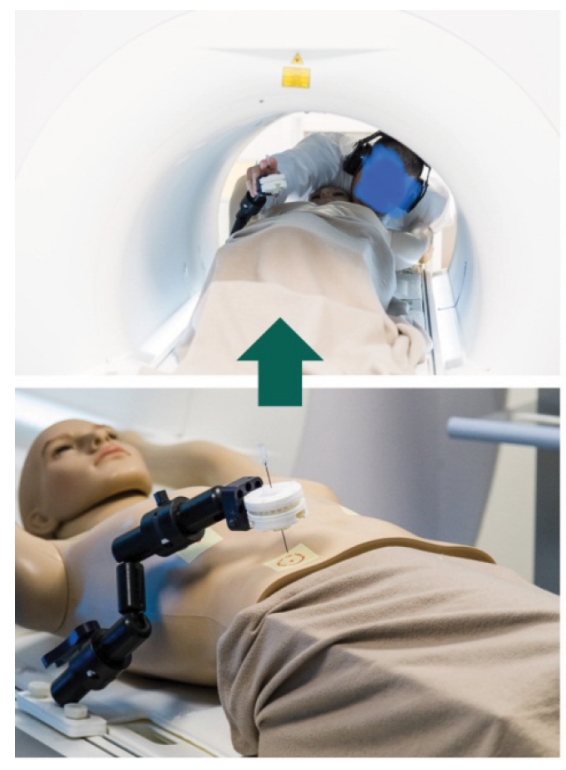

Alignment for paracentesis

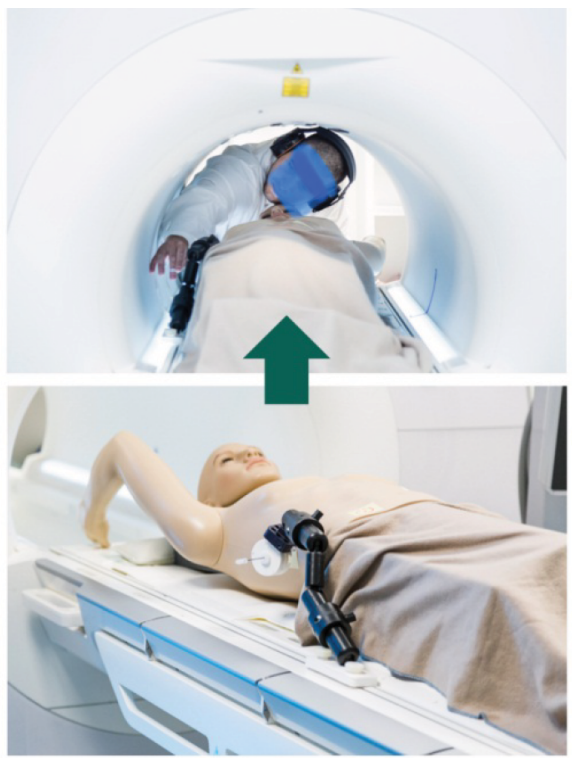

Alignment for liver or lung biospy

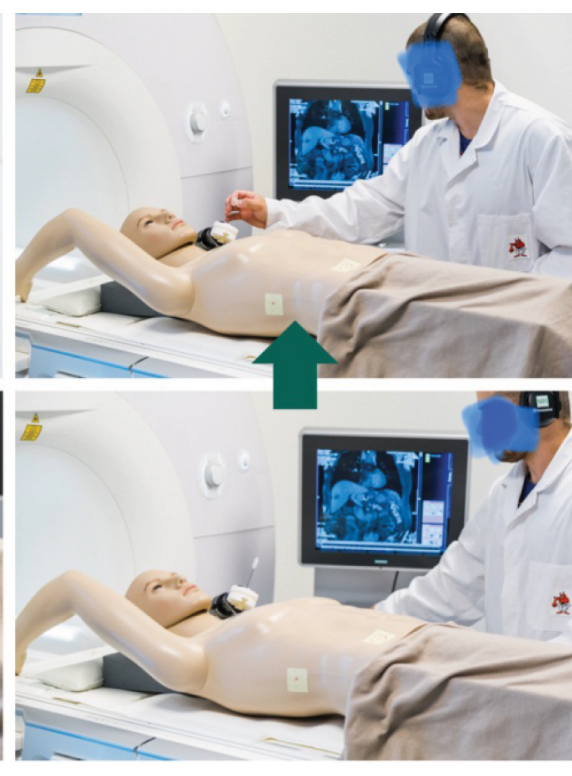

Alignment for thyroid biopsy

Figure 3 Typical horizontal-field $3 T$ system with $70 \mathrm{~cm}$ bore diameter.

Notes: Actual therapy is difficult for the surgeon due to an awkward position and also because only very little vertical space is available. It is typically at least $60 \mathrm{~cm}$ from the opening of the magnet bore to the center, which does not allow a direct view on the intervention area from outside. A holding arm could be very helpful (shown in black), but must be very small and flexible to fit into the magnet bore and to be of use for different interventional procedures.

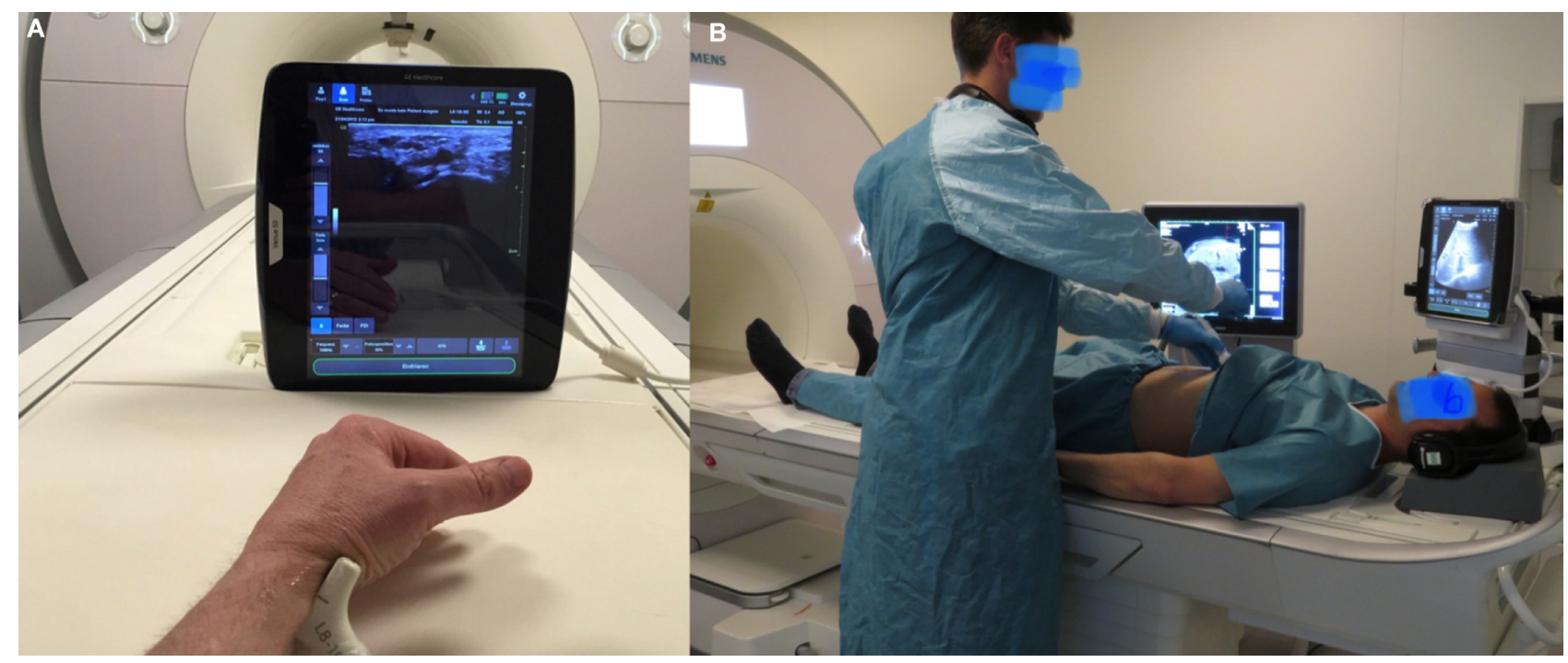

Figure 4 Ultrasound in a MRI imaging suite for MRI/US fusion.

Notes: (A) A tablet US of a large diagnostic imaging vendor placed only I m away from the 3T MRI of another large imaging vendor. The US system is not approved and certified for use in the MRI suite, but direct applications under US guidance could give a new boost to performing interventional and therapeutic procedures in the MRI suite. (B) Cognitive fusion of MRI and US images on separate monitors.

Abbreviations: MRI, magnetic resonance imaging; US, ultrasound.

This setup and process (Figure 5) allows a rather comfortable US-guided intervention for the surgeon standing next to the patient, with plenty of working space in all directions and excellent patient access directly on the MRI patient bed.

\section{Low-cost interventional MRI holding arm}

External intervention on the MRI patient bed may need additional MRI scanning during the procedure for verification or monitoring, which in turn requires that the therapeutic 


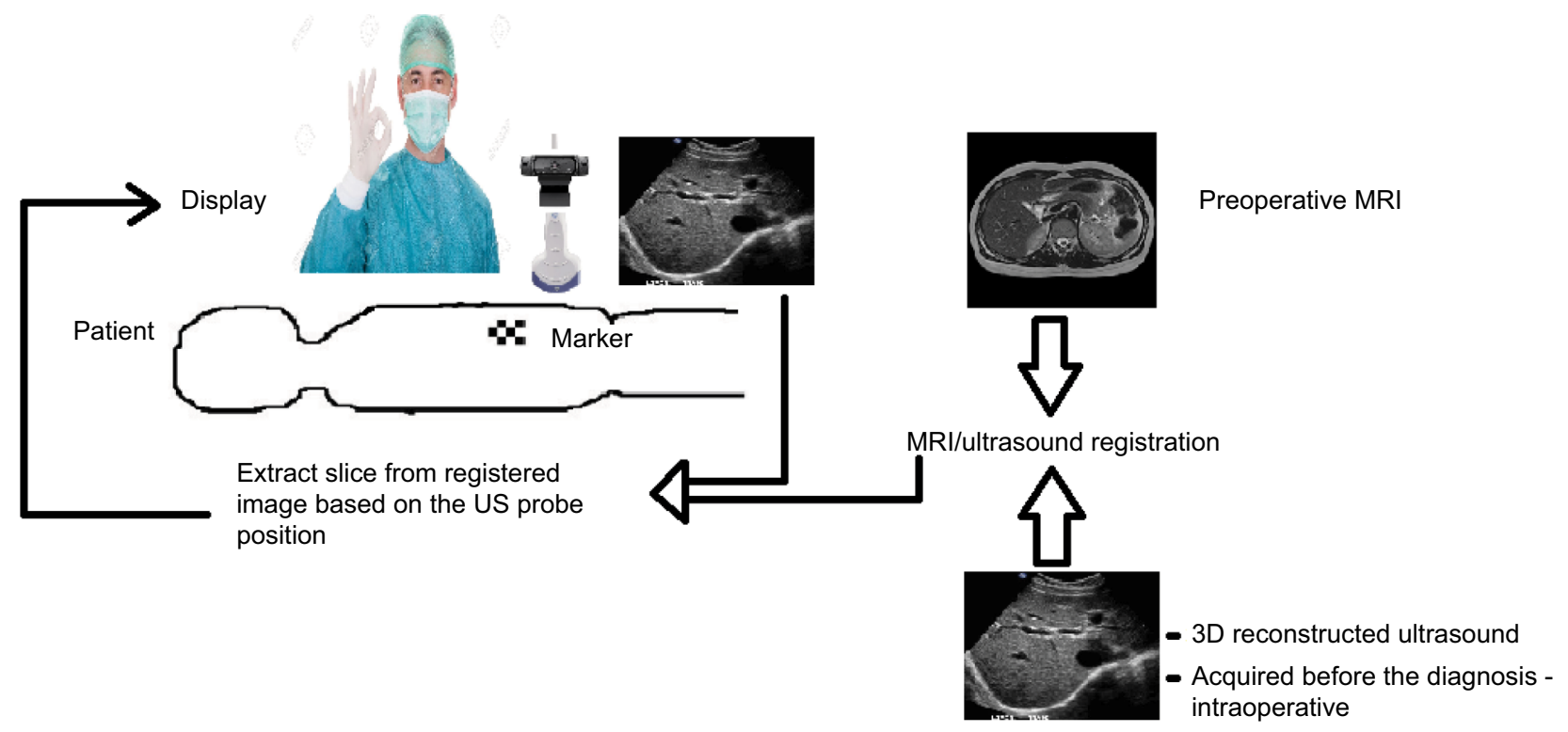

Figure 5 Concept of combining MRI images directly with US inside the MRI suite.

Notes: For this, markers on the patient are used as a small camera system that is directly mounted to the US probe. The live US is then registered to the MRI image and the actual procedure is performed under US guidance. If additional MRI imaging is required, then the patient is moved back into the magnet bore, and subsequently the US registered again to the new image data set.

Abbreviations: MRI, magnetic resonance imaging; US, ultrasound.

devices are fixed and held in position while the patient is moved back into the MRI magnet. This is ideally achieved using a completely MRI-compatible holding arm that is purely mechanical and manufactured from nonferrous or even plastic materials to avoid any magnetic attraction and to ensure that neither is the arm influenced by the MRI nor is the MRI negatively affected by the holding arm while maintaining a safe therapy setup for the patient.

We developed and 3D printed a completely new and modular holding arm concept from plastic material with 7 degrees of freedom that can be attached to the MRI bed railing and adjusted based on procedure needs and patient shape (Figure 6).

An initial MRI scan would provide the initial target site details used for the insertion path planning and the baseline scan for the US fusion.

Depending on the complexity, a quick MRI may need to be done to verify that the target site has been reached, and another scan can be done at the end of the procedure to inspect and record the therapy results (quality assurance).

\section{Discussion and conclusion}

MRI/US fused and used together and in parallel combines the excellent contrast and details of the MRI image with the real-time high-resolution device guidance of US and shifts the intervention space from inside the MRI to the MRI table.
Combining state-of-the-art high-field MRI with relatively low-cost US would provide superior baseline image quality with a fast and easy to use imaging system for guidance.

MRI/US fusion is already offered by several vendors for prostate biopsy applications. ${ }^{1,6}$ These systems generally, however, fuse preoperative MRI images with live and realtime US. Registration errors predominantly resulting from different patient position during imaging can be of the order of $7 \mathrm{~mm}$.

The presented setup showed the feasibility of using an US system as the primary guidance and monitoring device for interventional MRI procedures. To avoid the limitations of cognitive fusion and the cost and complexity of conventional optical tracking systems, we suggest a fusion of the MRI and US images using an inside-out tracking approach with a stereo camera system mounted to the US transducer camera in combination with MRI and US visible markers on the patient's body. Obviously when using this, the procedure should rather be called "Interventional US/MRI fusion procedure."

To make this procedure feasible for occasional use of the MRI system as an interventional and therapy guidance system, all the involved components need to be relatively inexpensive, easy to use, and easy to remove while providing the utmost safety for the patient and the surgeon.

For that, we propose the additional use of a small and very flexible holding arm that supports the surgeon and also holds 


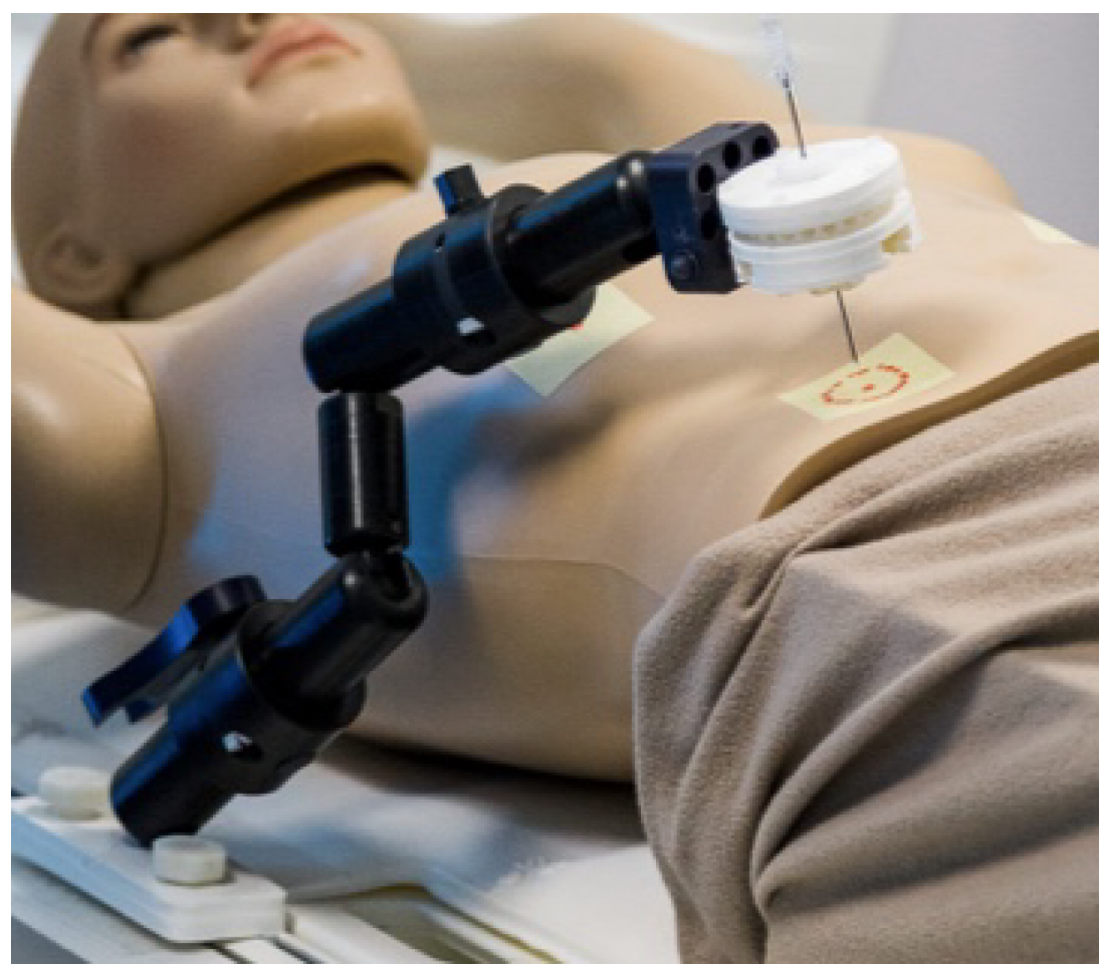

Figure $63 \mathrm{D}$ printed fully MRI-compatible modular holding arm individually adjustable for procedure needs and patient shape.

Notes: The arm with 7 degrees of freedom is directly attached to the rail of the bed and entirely manufactured from plastic materials.

Abbreviation: MRI, magnetic resonance imaging.

the therapy devices in place for additional MRI scanning during the procedure. This holding arm can be attached and removed in $<1$ minute. The US system can be moved to the end of the MRI bed (and back) in $<1$ minute, and the attached navigation camera on the US probe is permanently available. The time for the marker setup and the registration procedure is still a somewhat time-consuming process, but can certainly be automated and sped up in the very near future.

Since most MRI systems are almost exclusively used for diagnostic purposes, such an easy to set up and remove system would even be attractive for sites that only do few interventional or therapeutic MRI procedures. And, it would particularly be interesting for some of the easier and some of the more complex interventional MRI procedures (Figure 7 green and orange exclamation marks).

Difficult cardiac or neurosurgical procedures would require a dedicated surgery room with an MRI installed or available (Figure 1).

We evaluated the presented interventional MRI setups, conventional closed-bore high-field MRI (Figure 3), dedicated interventional MRI procedure room (Figure 1), and vertical-field MRI (Figure 2), with the proposed Interventional US/MRI fusion procedure setup (Figures 4-6) based on patient access, comfort to the surgeon, real-time imaging and therapy guidance, as well as total cost, for both easier and more advanced procedures (Figure 7).
The findings are presented in Table 1 and show that the Interventional US/MRI fusion procedure setup has potential advantages for all but the more complex procedures.

With MRI-compatible and approved US systems and easy to use and inexpensive accessories, interventional MRI could become an attractive option for other clinical applications as well.

If the interventional procedure is guided by the US system, an MRI-compatible in-room monitor is not needed anymore. As the US and the MRI are never used together, there will most likely not be any US-related MRI imaging artifacts. The cost of the in-room monitor alone is likely almost the same as the total cost of the devices mentioned in this paper.

This paper cannot present a thorough patient- and procedure-based evaluation research on the possible advantages of the setup as there are no MRI-compatible and MRI-safe US systems commercially available and as the holding arm and tracking method as presented are not clinically certified products. The intention of the presented perspective should help researchers rethink the current technical component research of interventional MRI.

We hope that many more research and industry teams will agree with this rethinking and help further develop procedures, tools, and devices that can be used for upcoming clinical validation. 


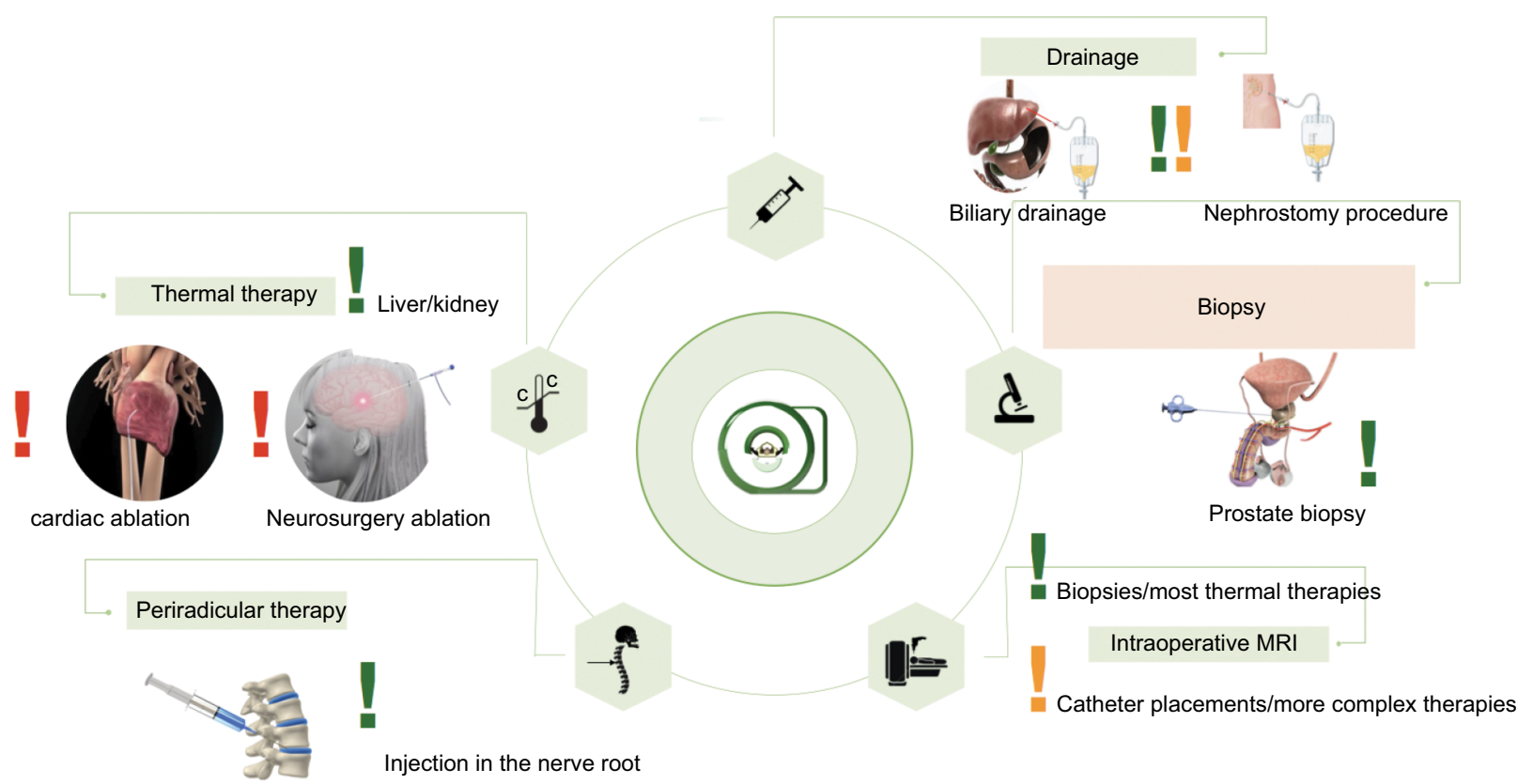

Figure 7 Possible therapies for the Interventional US/MRI fusion procedure setup.

Notes: The easier procedures are marked with a green exclamation mark. Medium/complex procedures (orange exclamation mark) could potentially be done with the proposed setup, while the difficult ones should be reserved to dedicated surgery rooms with advanced imaging modalities (eg, MRI) installed. The procedures marked with a red exclamation mark are reserved for dedicated interventional MRI suites as shown in Figure I.

Table I Comparison of different interventional MRI setups for easy and more complex procedures based on patient access and surgeon comfort, as well as on real-time therapy guidance capabilities in combination with high-quality imaging, and the ability to use for different procedures as highlighted in Figure 7.

\begin{tabular}{|c|c|c|c|c|}
\hline $\begin{array}{l}\text { Interventional } \\
\text { MRI issue }\end{array}$ & $\begin{array}{l}\text { Standard diagnostic MRI - } \\
\text { horizontal field (Figure 3) }\end{array}$ & $\begin{array}{l}\text { Standard diagnostic } \\
\text { MRI - vertical field } \\
\text { (Figure 2) }\end{array}$ & $\begin{array}{l}\text { In-room US fusion } \\
\text { added (Figure 4/5) }\end{array}$ & $\begin{array}{l}\text { Dedicated interventional } \\
\text { MRI suite (Figure I) }\end{array}$ \\
\hline $\begin{array}{l}\text { Patient access for the } \\
\text { therapist: How accessible } \\
\text { is the therapy region for } \\
\text { the surgeon? }\end{array}$ & $\begin{array}{l}- \\
\text { Typically } 70 \mathrm{~cm} \text { diameter and } \\
>60 \mathrm{~cm} \text { to the MRI center - } \\
\text { very difficult for the surgeon } \\
\text { to reach the surgical target. }\end{array}$ & $\begin{array}{l}0 \\
\text { Typically } 45 \mathrm{~cm} \text { vertical } \\
\text { opening and around } 50 \\
\mathrm{~cm} \text { to the MRI center, } \\
\text { patient access }>240 \\
\text { degrees. }\end{array}$ & $\begin{array}{l}+ \\
\text { Full patient access for } \\
\text { therapy. }\end{array}$ & $\begin{array}{l}+ \\
\text { Full patient access for } \\
\text { therapy. }\end{array}$ \\
\hline $\begin{array}{l}\text { Surgeon comfort: Is } \\
\text { the surgeon able to } \\
\text { perform the surgery in a } \\
\text { comfortable position? }\end{array}$ & $\begin{array}{l}\text { - } \\
\text { Surgeon has to reach into the } \\
\text { magnet bore in a sitting or } \\
\text { bent position. }\end{array}$ & $\begin{array}{l}0 \\
\text { Surgeon typically can sit } \\
\text { next to the magnet bore } \\
\text { but still has to reach in } \\
\text { the bore. }\end{array}$ & $\begin{array}{l}+ \\
\text { Surgeon can stand next } \\
\text { to the patient in a normal } \\
\text { standing position. }\end{array}$ & $\begin{array}{l}+ \\
\text { Surgeon can stand next } \\
\text { to the patient in a normal } \\
\text { standing position. }\end{array}$ \\
\hline $\begin{array}{l}\text { Real-time therapy } \\
\text { guidance: Focus is here on } \\
\text { whether real-time imaging } \\
\text { for tool and device } \\
\text { tracking is available - not } \\
\text { for the actual diagnostic } \\
\text { imaging process! }\end{array}$ & $\begin{array}{l}0 \\
\text { Only MRI is useable for } \\
\text { guidance - fast imaging is } \\
\text { low resolution; high-quality } \\
\text { imaging is slow. }\end{array}$ & $\begin{array}{l}-10 \\
\text { Only MRI is useable for } \\
\text { guidance - fast imaging } \\
\text { is low resolution; } \\
\text { high-quality imaging is } \\
\text { slow. Most vertical-field } \\
\text { systems are low field } \\
\text { (then -). }\end{array}$ & $\begin{array}{l}+ \\
\text { The US provides real-time } \\
\text { imaging to the previously } \\
\text { acquired high-quality MRI. } \\
\text { The image and system } \\
\text { fusion combines high- } \\
\text { quality imaging with real- } \\
\text { time imaging for therapy } \\
\text { guidance }\end{array}$ & $\begin{array}{l}+ \\
\text { Interventional MRI suites } \\
\text { come with additional real- } \\
\text { time imaging in form of } \\
\text { X-ray and/or US. }\end{array}$ \\
\hline $\begin{array}{l}\text { Interstitial therapies } \\
\text { (green “!” in Figure 7): Is } \\
\text { the system setup suitable } \\
\text { for the green therapies } \\
\text { listed in Figure 7? }\end{array}$ & $\begin{array}{l}0 \\
\text { Setup can be used with } \\
\text { limitations for easy } \\
\text { procedures. Very limited } \\
\text { space and reduced view of } \\
\text { surgical site. }\end{array}$ & $\begin{array}{l}+ \\
\text { Improved due to better } \\
\text { patient access and better } \\
\text { surgical site view. }\end{array}$ & $\begin{array}{l}++ \\
\text { Excellent patient access } \\
\text { in combination with real- } \\
\text { time image guidance and } \\
\text { no space restrictions in } \\
\text { the magnet bore. }\end{array}$ & $\begin{array}{l}++ \\
\text { Excellent patient access in } \\
\text { combination with real-time } \\
\text { image guidance and no } \\
\text { space restrictions in the } \\
\text { magnet bore. }\end{array}$ \\
\hline
\end{tabular}


Table I (Continued)

\begin{tabular}{|c|c|c|c|c|}
\hline $\begin{array}{l}\text { Interventional } \\
\text { MRI issue }\end{array}$ & $\begin{array}{l}\text { Standard diagnostic MRI - } \\
\text { horizontal field (Figure 3) }\end{array}$ & $\begin{array}{l}\text { Standard diagnostic } \\
\text { MRI - vertical field } \\
\text { (Figure 2) }\end{array}$ & $\begin{array}{l}\text { In-room US fusion } \\
\text { added (Figure 4/5) }\end{array}$ & $\begin{array}{l}\text { Dedicated interventional } \\
\text { MRI suite (Figure I) }\end{array}$ \\
\hline
\end{tabular}

More advanced therapies (orange and red “!” in Figure 7): Is the system setup suitable for the red! and orange therapies listed in Figure 7?

\begin{abstract}
MRI is not installed in a real surgical suite. Sterility and airflow is not acceptable for complicated open surgeries and there is only limited monitoring and support equipment available. Not feasible for complicated surgical procedures.
\end{abstract}

\section{0}

Cost of Setup for iMR is a conventional I.5T diagnostic MRI with a horizontal field
Cost of MRI plus $€ 50 \mathrm{k}$ to $€ I 50 \mathrm{k}$ for the Interventional MRI Package consisting of MRI-compatible in-room monitor(s), dedicated nonmagnetic therapy tools, anesthesia and monitoring equipment, and manipulation devices.

$\begin{array}{ll}\text { MRI is not installed in } & \text { MRI is not installed in } \\ \begin{array}{l}\text { a real surgical suite. } \\ \text { Sterility and airflow }\end{array} & \begin{array}{l}\text { a real surgical suite. } \\ \text { is not acceptable for }\end{array} \\ \begin{array}{ll}\text { complicated open } \\ \text { is not acceptable for }\end{array} \\ \begin{array}{ll}\text { only limited monitoring } \\ \text { and support equipment }\end{array} & \text { for surgeries that } \\ \text { available. Not feasible } & \text { require real-time therapy }\end{array}$

for complicated surgical procedures.

-

\section{Cost of a high-field} vertical-field $M R I$ is about $€ 300 \mathrm{k}$ to $€ 500 \mathrm{k}$ above the price for a standard diagnostic MRI with horizontal field plus the cost of the Interventional MRI Package.
0

Cost of MRI plus Interventional Package plus $€ 50.000$ for the US and tracking hardware and software.
$+$ MRI is installed in a real surgical suite and comes with all equipment found in an advanced surgical setup.
$-$

The cost for the building/ construction and the additional equipment are very high ( $>€ 1.000 \mathrm{k}$ ) also a reason why there are only few such systems installed worldwide

Notes: The additional cost for such a setup was also coarsely evaluated. The table evaluation is always based upon the best possible option for the particular point in question (eg, the most open patient access for therapeutic interaction is a patient on a bed freely accessible, which gives a " + " to the "In-Room US Fusion" concept and the "Dedicated Interventional MRI"). A "0" indicates it is possible/OK a "-" not feasible, "+" feasible, and "++"|"--" excellent/prohibitive. Abbreviations: MRI, magnetic resonance imaging; iMRI, interventional MRI; US, ultrasound.

\section{Disclosure}

The authors report no conflicts of interest in this work.

\section{References}

1. Logan JK, Rais-Bahrami S, Turkbey B, et al. Current status of MRI and ultrasound fusion software platforms for guidance of prostate biopsies. BJU Int. 2014;114(5):641-652.

2. Grönemeyer DHW, Seibel RMM, Melzer A, et al. Future of advanced guidance techniques by interventional CT and MRI. Minim Invas Ther. 1995;4:251.

3. Krombach GA, Schmitz S, Friebe M, et al. Interventionelle MRT bei 3 Tesla: Durchführbarkeit endovaskulärer Interventionen. RöFo. $2008 ; 180$

4. Balakrishnan S, Friebe M. Real-time MRI/US fusion using inside-out tracking of virtually generated markers (ORtoMVM). In: CARS 2017 Computer Assisted Radiology and Surgery - 31st International Congress and Exhibition; June 20-24, 2017; Barcelona.
5. Conley RH, Meszoely IM, Pheiffer TS, Weis JA, Yankeelov TE, Miga MI. Image to physical space registration of supine breast MRI for image guided breast surgery. In: Proceedings Volume 9036, Medical Imaging 2014: Image-Guided Procedures, Robotic Interventions, and Modeling; 2014; San Diego, CA.

6. Gayet M, van der Aa A, Beerlage HP, Schrier BP, Mulders PF, Wijkstra $\mathrm{H}$. The value of magnetic resonance imaging and ultrasonography (MRI/ US)-fusion biopsy platforms in prostate cancer detection: a systematic review. BJU Int. 2016;117(3):392-400.

7. Krug J, Boese A, Odenbach R, Friebe M . Quick and easy: iMRI procedure improvement using non-registered portable US. Presented at: 11th Interventional MRI Symposium; October 7-8, 2016; Baltimore, MA.

8. Nagaraj Y, Benedicks C, Matthies P, Friebe M. Advanced inside-out tracking approach for real-time combination of MRI and US images in the radio-frequency shielded room using combination markers. Conf Proc IEEE Eng Med Biol Soc. 2016;2016:2558-2561.

9. Matthies P, Okur A, Wendler T, Navab N, Friebe M. Combination of intraoperative freehand SPECT imaging with MR images for guidance and navigation. Conf Proc IEEE Eng Med Biol Soc. 2013;2013:3383-3386.
Medical Devices: Evidence and Research

\section{Publish your work in this journal}

Medical Devices: Evidence and Research is an international, peerreviewed, open access journal that focuses on the evidence, technology, research, and expert opinion supporting the use and application of medical devices in the diagnosis, monitoring, treatment and management of clinical conditions and physiological processes. The identification of novel

\section{Dovepress}

devices and optimal use of existing devices which will lead to improved clinical outcomes and more effective patient management and safety is a key feature. The manuscript management system is completely online and includes a quick and fair peer-review system. Visit http://www. dovepress.com/testimonials.php to read real quotes from authors. 